\title{
Clinical considerations and treatment options in Staphylococcus aureus foot infections
}

\author{
Catalin Bejinariu', Carmen Giuglea ${ }^{2,3}$, Silviu Marinescu ${ }^{1,3}$ \\ ${ }^{1}$ Department of Plastic and Reconstructive Surgery, \\ "Bagdasar-Arseni" Emergency Clinical Hospital, Bucharest, Romania \\ ${ }^{2}$ Department of Plastic and Reconstructive Surgery, \\ "Sf. Ioan" Emergency Clinical Hospital, Bucharest, Romania \\ 3"Carol Davila" University of Medicine and Pharmacy, Bucharest, Romania
}

\begin{abstract}
Objectives. Foot cellulitis caused by Staphylococcus aureus raises multiple problems regarding early diagnosis and choice of the optimal treatment, especially in diabetic patients. The research aims to research clinical and prognostic features related to patients diagnosed with foot cellulitis.

Material and methods. The study presents the clinical and prognostic features of 41 patients who received treatment at the "Bagdasar-Arseni" Emergency Clinical Hospital.

Outcomes. The results of the analysis show that cellulitis caused by Staphylococcus aureus is associated with a favorable prognosis in the case of the rapid establishment of specific antibiotic treatment in combination with serial excisional debridement.

Conclusions. It is well known that the late establishment of therapeutic measures can have devastating consequences, culminating in the death of the patient. However, it should not be neglected that the combination of the surgical treatment with the specific antibiotic therapy can most often be the salvage solution for these difficult cases.
\end{abstract}

Keywords: diabetic foot, Staphylococcus aureus infection, foot cellulitis

\section{INTRODUCTION}

Extensive diabetic foot infections often require amputation, a therapeutic approach frequently associated with the psychological preparation of the patients, as well as with a marked limitation of the patients' working capacity. The major risk of developing a generalized infection following the conservative approach is considered by most surgeons to be unacceptable. Therefore, an increasing number of patients benefit from surgical treatment consisting of amputation from the upper third of the leg $(1,2)$. In this context, the present study aims to identify the possible surgical approaches that, for increased safety purposes, could significantly re- duce the rate of amputations in hospitalized patients for the treatment of cellulitis caused by Staphylococcus aureus.

\section{MATERIAL AND METHODS}

Data obtained related to the treatment of foot infections was collected from 41 patients admitted to the Plastic Surgery Department of the "BagdasarArseni" Emergency Clinical Hospital in the period January 2015 - August 2019.

The inclusion criteria were represented by:

- age between 18 and 70 years;

- positive cultures for Staphylococcus aureus; 
- obtaining the agreement on performing the customized surgical treatment;

- the informed consent related to the use of medical information for scientific purposes.

The exclusion criteria were represented by:

- age below 18 years or over 70 years;

- psychic or psychological context with impact on the patient's discernment;

- the disagreement related to data processing for scientific purposes.

The surgical treatment involved daily serial excisions, with the removal of the devitalized tissues, while respecting the integrity of the viable anatomical structures, so as to create the premises of harmonious healing, without the large, non-selective excision of the tissues at this level. In order to perform the initial excision, spinal anesthesia was performed, followed in the next stages of the treatment by serial excisions performed under local anesthesia or, in selected cases, without anesthesia (severe neuropathy). The coverage of the post-excisional defects was made by plasties with free split skin harvested from the anterior face of the thigh.

The antibiotic treatment was instituted starting with the first day of hospitalization, by administering the broad spectrum therapy until the result of the antibiogram was obtained. To achieve this goal, the combination of Ceftriaxone, Gentamicin and Metronidazole was used.

In order to control the glucose values in the patients with high fluctuations, an interdisciplinary diabetes and nutrition diseases consult was requested, followed by telephone counseling in order to continue the appropriate therapy.
In order to quantify the quality of the patients' experience with regard to the treatment and the postoperative result, the patients' feedback was requested using grades from 1 to 10,1 corresponding to the lowest satisfaction and 10 to the highest satisfaction, a method that allowed an objective analysis of the patient satisfaction regarding this type of therapeutic approach.

\section{RESULTS}

The results of the study show that $92.68 \%$ of the general group of patients benefited from serial surgical excision that resulted in the healing of the lesions, the amputation of the foot being required in 3 cases. In $23.80 \%$ of the cases it was necessary to perform digital amputations, followed by serial excision.

From the general group of 41 patients, 31 patients had a personal history of metabolic pathology (type I or II diabetes), 7 patients were diagnosed in the context of performing the treatment of foot cellulitis, while 3 patients did not show changes in blood glucose levels during hospitalization.

The average hospitalization time was 15.7 days, being strongly influenced by the large number of diabetic patients included in the study, the delayed healing characteristic related to these patients contributing significantly to the increase of the average duration of hospitalization (Figure 1).

Regarding the dressings changes, it has been shown that in the first 3 days after admission, changing two bandages per day is salutary due to the strongly exudative phase of the injuries. The
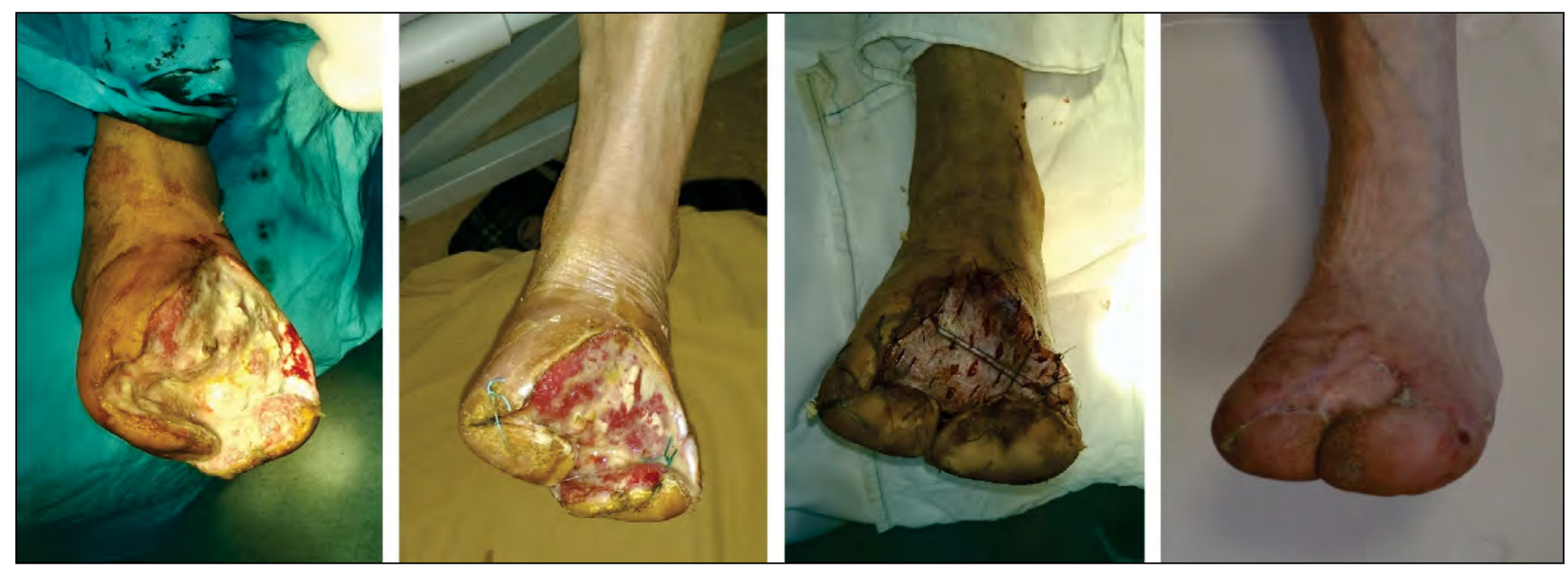

FIGURE 1. Foot infections - post-excisional defect followed by reconstruction 
advantages of this approach are: removal of pathological secretions from the lesion, reduction of bacterial contamination and increased exposure time to the antibacterial substances included in the dressing protocol.

\section{DISCUSSIONS}

In most cases, the treatment of foot cellulitis requires a multidisciplinary approach. Frequently patients suffering from septic processes affecting the pelvic limbs associate metabolic imbalances and cardiovascular disorders that reduce the available therapeutic options $(3,4,5)$.

In the absence of comorbidities, the lower limb cellulitis caused by Staphylococcus aureus is associated with a favorable evolution after the surgical treatment combined with the specific antibiotic therapy. Incisions performed for the evacuation of wound secretions, followed by serial excisions contribute significantly to the healing of septic outbreaks. However, late diagnosis caused by the delayed hospital admissions for patients with limited health education may lead to the loss of the affected limb.

Cellulitis occurring in the diabetic context is characterized by a delayed evolution that often requires the execution of serial excisions for long periods of time, the deepening of the lesions and the extension of the bacterial contamination being often issues for these patients $(6,7,8)$. Initial infections caused by Staphylococcus aureus often evolve into complex cellulitis with multiple pathogens, Pseudomonas aeruginosa and Enterobacter being frequently identified in these patients' cultures $(9,10)$. Broad-spectrum antibiotic treatment instituted immediately after performing the wound secretion examination is an essential element in the treatment of these patients. Subsequently, the combination of the surgical treatment with the specific antibiotic therapy points the patient towards a favorable evolution, which is often marked by relapses and exacerbations that require additional excisions and even amputations $(11,12,13)$.

Infections that occur in patients with obliterating arteriopathy are in most cases associated with an unfavorable prognosis, in this respect efficient communication with the patient characterized by the presentation of all the therapeutic options, as well as discussing the increased risk of amputation are essential elements in the therapeutic protocol.

Regarding the communication between the doctor and the patient, an important element that contributes to the increase of the adherence to the treatment is represented by the detailed presentation of the risks involved related to not following the doctor's recommended conduct, as well as by the delayed treatment $(14,15)$. The imminence of the amputation increases the degree of compliance related to these patients, contributing significantly to the adherence to the treatment and following the indications of the medical staff.

The patient satisfaction related to the postoperative results is in most cases high in these cases, the clinical aspect specific to this type of pathology contributing significantly to the increased degree of awareness regarding the severity of the lesions. Therefore, the surgical treatment, even in cases where it is necessary to perform plasties with the free split skin graft, is associated with an increased level of patient satisfaction.

\section{CONCLUSIONS}

Serial excision of devitalized tissues in patients diagnosed with foot cellulitis induced by Staphylococcus aureus represents a firm solution for solving these cases and is a solid alternative to the classic surgical treatment, characterized by radical excisions that often point the therapeutic protocol in the direction of performing amputations.

Increasing the frequency of dressings in the exudative stage contributes significantly to reducing the hospitalization period and accelerating the healing by: removing pathological secretions, reducing local contamination and increasing the duration of exposure to the applied antibacterial substances.

Effective communication with the patients and the detailed presentation of the risks contribute significantly to increasing the patients' adherence to treatment and improving their satisfaction related to the postoperative results.

Conflict of interest: none declared Financial support: none declared 


\section{REFERENCES}

1. Abu Naser SS, Mahdi AO. A proposed expert system for foot diseases diagnosis. American Journal of Innovative Research and Applied Sciences. 2016; 2(4):160-173.

2. Jain AKC. Type 1 diabetic foot complications. The Journal of Diabetic Foot Complications, 2016;8(1):17-22.

3. Raff AB, Kroshinsky D. Cellulitis A Review. JAMA. 2016; 316(3):325-337.

4. Esposito S, Noviello S, De Caro F. New insights into classification, epidemiology and microbiology of SSTIs, including diabetic foot infections. Le Infezioni in Medicina. 2018;1:3-14.

5. Cervantes-García E, García-González R, Reséndiz-Albor A et al. Infections of Diabetic Foot Ulcers with Methicillin-Resistant Staphylococcus aureus. The International Journal of Lower Extremity Wounds. Int J Low Extrem Wounds. 2015;14(1):44-9.

6. Hale AJ, Vicks E, LaSalvia MT et al. Methicillin-Resistant Staphylococcus aureus Endocarditis from a Diabetic Foot Ulcer. Journal of the American Podiatric Medical Association. 2018;108(6):528-531.

7. El-Sayed D, Nouvong A, Carlson P et al. Bacteriology of Infected, Subcutaneous Tissue in Diffuse Cellulitis and Foot Ulcer Infections in Patients With Diabetes Mellitus Using Cultures and Molecular Identification Techniques. Open Forum Infectious Diseases. 2015; 2(1), 1524.

8. Dunyach-Remy C, Essebe CN, Sotto A et al. Staphylococcus aureus Toxins and Diabetic Foot Ulcers: Role in Pathogenesis and Interest in Diagnosis. Toxins. 2016;8(7):209.
9. Bejinariu C, Marinescu S, Giuglea C. Studiu asupra reconstrucţiei de sân după mastectomie la pacientele diabetice. Romanian Medical Journal. 2019; 66(1):50-54.

10. Lipsky BA, Berendt AR, Cornia PB et al. 2012 Infectious Diseases Society of America Clinical Practice Guideline for the Diagnosis and Treatment of Diabetic Foot Infections. Clinical Infectious Diseases. 2012; 54(12):e132-e173.

11. Petrakis I, Kyriopoulos IJ, Ginis A et al. Losing a foot versus losing a dollar; a systematic review of cost studies in diabetic foot complications. Expert Review of Pharmacoeconomics \& Outcomes Research. 2017;17(2):165-180.

12. Dhamodharan U, Teena R, Kumar RV et al. Circulatory levels of B-cell activating factor of the TNF family in patients with diabetic foot ulcer: Association with disease progression. Wound Repair and Regeneration. 2019; 27(5):442-449.

13. Samsell B, McLean J, Cazzell S et al. Health economics for treatment of diabetic foot ulcers: A cost-effectiveness analysis of eight skin substitutes. Journal of Wound Care. 2019; 28(9):S14-S26.

14. Chastain CA, Klopfenstein N, Serezani CH et al. A Clinical Review of Diabetic Foot Infections. Clinics in Podiatric Medicine and Surgery. 2019; 36(3):381-395.

15. Chatman BC, Parks VE. Bone Reconstruction in the Diabetic Foot. Clinics in Podiatric Medicine and Surgery. 2019; 36(3):457-468. 